\title{
Abelian Decomposition and Monopole Condensation in QCD
}

\author{
Y. M. Cho ${ }^{1,2,3, a}$ \\ ${ }^{1}$ Administration Building 310-4, Konkuk University, Seoul 143-701, Korea \\ ${ }^{2}$ Center for Quantum Spacetime, Sogang University, Seoul 04107, Korea \\ ${ }^{3}$ School of Physics and Astronomy, Seoul National University, Seoul 151-747, Korea
}

\begin{abstract}
We demonstrate the monopole condensation in QCD using the Abelian decomposition. The Abelian decomposition decomposes the gluons to the color neutral binding gluons (the neurons and the monopoles) and the colored valence gluons (the chromons), and shows that QCD can be viewed as the restricted QCD (RCD) made of the binding gluons which has the chromons as colored source. This simplifies the QCD dynamics greatly. In the perturbative regime this decomposes the gluon propagater to the neuron propagaters and the chromon propagaters, and simplifies the Feynman diagram. In the non-perturbative regime this allows us to calculate the QCD effective potential gauge independently, and demonstrate the monopole condensation unambiguously.
\end{abstract}

\section{Introduction}

One of the most challenging problems in theoretical physics is the confinement problem in quantum chromodynamics (QCD). Two outstanding conjectures of the confinement mechanismare the monopole condensation and the Abelian dominance [1-5]. It has long been argued that the confinement in QCD can be triggered by the monopole condensation [1-3]. Indeed, if one assumes the monopole condensation, one can easily argue that the ensuing dual Meissner effect could guarantee the color confinement. To prove the monopole condensation, however, has been extremely difficult.

A natural way to establish the monopole condensation in QCD is to show that the quantum fluctuation triggers a phase transition similar to the dimensional transmutation observed in massless scalar QED [6]. There have been many attempts to demonstrate this. Savvidy has first calculated the effective action of SU(2) QCD in the presence of an ad hoc color magnetic background, and has almost "proved" the magnetic condensation which is known as the Savvidy vacuum [7].

Unfortunately, the subsequent calculation repeated by Nielsen and Olesen showed that the effective action has an extra imaginary part which destablizes the magnetic condensation. This is known as the "Savvidy-Nielsen-Olesen (SNO) instability" [8-10]. The origin of this instability can be traced to the tachyonic modes in the functional determinant of the gluon loop integral.

\footnotetext{
ae-mail: ymcho7@konkuk.ac.kr
} 
But in physics we encounter the tachyons when we do something wrong. For example, in spontaneous symmetry breaking we have tachyons when we choose the false vacuum. Similarly, in NeveuSchwarz-Ramond (NSR) string theory we have the tachyonic vacuum when we do not make the theory supersymmetric and modular invariant with the Gliozzi-Scherk-Olive (GSO) projection [11, 12]. The question here is how to remove the tachyonic modes in the gluon functional determinant, and how to justify that.

We emphasize, however, that the most seriuos defect of the SNO vacuum is not that it is unstable but that it is not gauge invariant. So even if the Savvidy vacuum were made stable, it can not be the QCD vacuum. Because of this Nielsen and Olesen has proposed the so-called "Copenhagen vacuum", the randomly oriented piecewise Savvidy vacuum [8]. But one can not obtain a gauge invariant vacuum randomly orienting something which is not gauge invariant.

On the other hand the Abelian dominance proposed by 'tHooft asserts that the "Abelian part" of QCD must be responsible for the confinement $[4,5]$. The justification of this is that the colored (non-Abelian) gluons can not play any role in the confinement, because they (just like the quarks) themselves have to be confined. So, only the neutral (Abelian) gluons, if at all, could possibly contribute to the confinement. In this sense, this conjecture must be true. In fact we can show explicitly that the Abelian part of the potential is responsible for the confining force in QCD [13].

The Abelian dominance has become very popular and widely been studied in lattice QCD [5]. However, it has serious drawbacks. The popular way to obtain the Abelian part with "the maximal Abelian gauge" is gauge dependent. More seriously, this does not tell exactly what constitutes the Abelian part. Obviously the Abelian part must contain the trivial Maxwell-type Abelian potential, but this $\mathrm{U}(1)$ potential is not supposed to generate the confinement. So the Abelian part must contain something else, but the Abelian dominance does not specify what is that. So, even if we prove the Abelian dominance, we can not tell what is really responsible for the confinement.

The gauge independent Abelian projection defined by the magnetic isometry can cure this defect. It tells that the Abelian potential is made of two parts, the non-topological Maxwell part and the topological monopole part [2,3]. So this allows us to test not only the Abelian dominance but also the monopole condensation gauge independently. With this we can pinpoint exactly what is responsible for the confinement. In this talk I discuss how we can demonstrate the monopole condensation in QCD using the Abelian decomposition.

It has generally been believed that the non-Abelian gauge symmetry is so tight that it defines the theory almost uniquely. Moreover, the gauge symmetry binds different gauges so strongly that the theory does not allow any simplification. The Abelian decomposition tells that this popular wisdom is wrong.

The Abelian decomposition decomposes the gluons (in general the non-Abelian gauge potentials) to two different types, the color neutral binding gluons called the neurons and the colored valence gluons called the chromons, gauge independently. This is very important in hadron spectroscopy because the chromons, just like the quarks, can form the color singlet bound states and become the constituent of hadrons. This generalizes the quark model to the quark and chromon model. In particular, this plays the essential role for us to understand the glueball spectrum and its mixing with quarkonium.

As importantly, it tells that we can have simpler QCD called the restricted QCD (RCD) made of the binding gluons which nevertheless has the full non-Abelian gauge symmetry. Moreover, QCD can be interpreted as RCD which has the chromons as colored source. This greatly simplifies the dynamics of QCD, in general the non-Abelian gauge theory. 
Furthermore, the Abelian decomposition separates the non-Abelian monopole gauge independently, and allows us to prove the confining potential in QCD comes from the monopole. In fact it allows us to calculate the effective potential of QCD and demonstrates that QCD vacuum is made of the monopole condensation. In this talk we discuss the Abelian decomposition of QCD and show how it can be used to demonstrate the monopole condensation gauge independently.

\section{Abelian decomposition: A Review}

Let us review the Abelian decomposition first. Conside the SU(2) QCD for simplicity, and let $\left(\hat{n}_{1}, \hat{n}_{2}, \hat{n}_{3}=\hat{n}\right)$ be an arbitrary right-handed local orthonormal basis. To make the Abelian decomposition we choose any direction, to be explicit $\hat{n}$, to be the Abelian direction and impose the isometry to project out the restricted potential $\hat{A}_{\mu}$ which describes the Abelian sub-dynamics of QCD [2,3]

$$
\begin{gathered}
D_{\mu} \hat{n}=\left(\partial_{\mu}+g \overrightarrow{A_{\mu}} \times\right) \hat{n}=0, \\
\vec{A}_{\mu} \rightarrow \hat{A}_{\mu}=A_{\mu} \hat{n}-\frac{1}{g} \hat{n} \times \partial_{\mu} \hat{n}=\mathcal{A}_{\mu}+C_{\mu}, \\
\mathcal{A}_{\mu}=A_{\mu} \hat{n}, \quad C_{\mu}=-\frac{1}{g} \hat{n} \times \partial_{\mu} \hat{n}, \quad A_{\mu}=\hat{n} \cdot \overrightarrow{A_{\mu}} .
\end{gathered}
$$

This is the Abelian projection which projects out the color neutral binding gluons.

Notice the followings. First, $\hat{A}_{\mu}$ is precisely the connection which leaves the Abelian direction invariant under the parallel transport (which makes $h n$ covariantly constant). Second, it is made of two parts, the topological (Diracian) $C_{\mu}$ which describes the non-Abelian monopole and the nontopological (Maxwellian) $\mathcal{A}_{\mu}$. Third, the decomposition is gauge independent because $\hat{n}$ is arbitrary. We can rotate $\hat{n}$ to any direction and still get exactly the same decomposition. This is the most important point.

With this we have

$$
\begin{gathered}
\hat{F}_{\mu \nu}=\left(F_{\mu \nu}+H_{\mu \nu}\right) \hat{n}=G_{\mu v} \hat{n}, \\
F_{\mu \nu}=\partial_{\mu} A_{v}-\partial_{\nu} A_{\mu} \\
H_{\mu \nu}=\partial_{\mu} C_{v}-\partial_{\nu} C_{\mu}, \quad C_{\mu}=-\frac{1}{g} \hat{n}_{1} \cdot \partial_{\mu} \hat{n}_{2}, \\
G_{\mu \nu}=\partial_{\mu} B_{v}-\partial_{\nu} B_{\mu}, \quad B_{\mu}=A_{\mu}+C_{\mu} .
\end{gathered}
$$

This tells two things. First, $\hat{F}_{\mu \nu}$ has only the Abelian component. Second, $\hat{F}_{\mu \nu}$ is made of two potentials, the electric (non-topological) $A_{\mu}$ and magnetic (topological) $C_{\mu}$.

With (1) we can recover the full QCD potential adding the non-Abelian (colored) part $\vec{X}_{\mu}[2,3]$

$$
\begin{gathered}
\vec{A}_{\mu}=\hat{A}_{\mu}+\vec{X}_{\mu}, \\
\vec{X}_{\mu}=\frac{1}{g} \hat{n} \times D_{\mu} \hat{n}, \quad \hat{n} \cdot \vec{X}_{\mu}=0 .
\end{gathered}
$$

Under the infinitesimal gauge transformation

$$
\delta \overrightarrow{A_{\mu}}=\frac{1}{g} D_{\mu} \vec{\alpha}, \quad \delta \hat{n}_{i}=-\vec{\alpha} \times \hat{n}_{i}
$$


we have

$$
\begin{gathered}
\delta \hat{A}_{\mu}=\frac{1}{g} \hat{D}_{\mu} \vec{\alpha}, \quad\left(\hat{D}_{\mu}=\partial_{\mu}+g \hat{A}_{\mu} \times\right), \\
\delta A_{\mu}=\frac{1}{g} \hat{n} \cdot \partial_{\mu} \vec{\alpha}, \quad \delta C_{\mu}=-\frac{1}{g} \hat{n} \cdot \partial_{\mu} \vec{\alpha}, \\
\delta \vec{X}_{\mu}=-\vec{\alpha} \times \vec{X}_{\mu} .
\end{gathered}
$$

This tells that $\hat{A}_{\mu}$ has the full SU(2) gauge degrees of freedom, even though it is restricted. Moreover, this confirms that $\vec{X}_{\mu}$ becomes gauge covariant. This is a direct consequence of the fact that the connection space (the space of all potentials) forms an affine space.

This is the Abelian decomposition which decomposes the gluons to the color neutral binding gluons and the colored valence gluons gauge independently. We can express the Abelian decomposition graphically. This is shown in Fig. 1, where the gluons are decomposed to the binding gluons and the valence gluons in (A), and the binding gluons are decomposed further to the non-topological Maxwell part $\mathcal{A}_{\mu}$ and the topological Dirac part $C_{\mu}$ in (B). In the literature this is known as Cho decomposition, Cho-Duan-Ge (CDG) decomposition, or Cho-Faddeev-Niemi (CFN) decomposition [14-16].

From this we can construct RCD which has the full non-Abelian gauge symmetry but is simpler than the QCD

$$
\begin{gathered}
\mathcal{L}_{R C D}=-\frac{1}{4} \hat{F}_{\mu \nu}^{2}=-\frac{1}{4} F_{\mu \nu}^{2} \\
+\frac{1}{2 g} F_{\mu \nu} \hat{n} \cdot\left(\partial_{\mu} \hat{n} \times \partial_{\nu} \hat{n}\right)-\frac{1}{4 g^{2}}\left(\partial_{\mu} \hat{n} \times \partial_{\nu} \hat{n}\right)^{2},
\end{gathered}
$$

which describes the Abelian subdynamics of QCD. Since RCD contains the non-Abelian monopole degrees explicitly, it provides an ideal platform for us to study the monopole dynamics gauge independently.

From (3) we have

$$
\vec{F}_{\mu v}=\hat{F}_{\mu v}+\hat{D}_{\mu} \vec{X}_{v}-\hat{D}_{v} \vec{X}_{\mu}+g \vec{X}_{\mu} \times \vec{X}_{v}
$$

With this we can express QCD by

$$
\begin{gathered}
\mathcal{L}_{Q C D}=-\frac{1}{4} \vec{F}_{\mu \nu}^{2}=-\frac{1}{4} \hat{F}_{\mu \nu}^{2}-\frac{1}{4}\left(\hat{D}_{\mu} \vec{X}_{v}-\hat{D}_{v} \vec{X}_{\mu}\right)^{2} \\
-\frac{g}{2} \hat{F}_{\mu \nu} \cdot\left(\vec{X}_{\mu} \times \vec{X}_{v}\right)-\frac{g^{2}}{4}\left(\vec{X}_{\mu} \times \vec{X}_{v}\right)^{2} .
\end{gathered}
$$

This is the extended QCD (ECD) which confirms that QCD can be viewed as RCD made of the binding gluons, which has the colored valence gluons as its source [2,3]. Notice that, although mathematically ECD is identical to QCD, physically it aquires a totally different meaning.

\section{Abelian Decomposition of SU(3) QCD}

The Abelian decomposition of SU(3) QCD is a bit more complicated, but is well known. Since SU(3) has rank two, we have two Abelian subgroups in $\operatorname{SU}(3)$. Let $\hat{n}_{i}(i=1,2, \ldots, 8)$ be the local orthonormal 


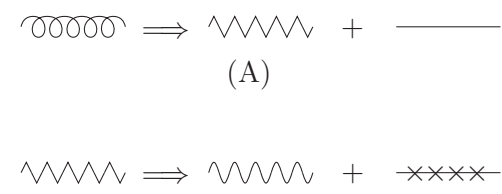

(B)

Figure 1. The Abelian decomposition of the gluons. The gluon is decomposed to the binding gluon (kinked line) and the valence gluon (straight line) in (A), and the binding gluon is further decomposed to the Maxwell part (wiggly line) and Dirac part (spiked line) in (B).

$\mathrm{SU}(3)$ basis. Clearly we can choose the Abelian directions to be $\hat{n}_{3}=\hat{n}$ and $\hat{n}_{8}=\hat{n}^{\prime}$. Now make the Abelian projection by

$$
D_{\mu} \hat{n}=0
$$

This automatically guarantees [17]

$$
D_{\mu} \hat{n}^{\prime}=0, \quad \hat{n}^{\prime}=\frac{1}{\sqrt{3}} \hat{n} * \hat{n}
$$

where $*$ denotes the $d$-product. This is because $\mathrm{SU}(3)$ has two vector products, the anti-symmetric $f$-product and the symmetric $d$-product.

Solving (9), we have the following Abelian projection which projects out two neutral binding gluons,

$$
\begin{gathered}
\overrightarrow{A_{\mu} \rightarrow \hat{A}_{\mu}=} A_{\mu} \hat{n}+A_{\mu}^{\prime} \hat{n}^{\prime}-\frac{1}{g} \hat{n} \times \partial_{\mu} \hat{n}-\frac{1}{g} \hat{n}^{\prime} \times \partial_{\mu} \hat{n}^{\prime} \\
=\sum_{p} \frac{2}{3} \hat{A}_{\mu}^{p}, \quad(p=1,2,3), \\
\hat{A}_{\mu}^{p}=A_{\mu}^{p} \hat{n}^{p}-\frac{1}{g} \hat{n}^{p} \times \partial_{\mu} \hat{n}^{p}=\mathcal{A}_{\mu}^{p}+C_{\mu}^{p}, \\
A_{\mu}^{1}=A_{\mu}, \quad A_{\mu}^{2}=-\frac{1}{2} A_{\mu}+\frac{\sqrt{3}}{2} A_{\mu}^{\prime}, \\
A_{\mu}^{3}=-\frac{1}{2} A_{\mu}-\frac{\sqrt{3}}{2} A_{\mu}^{\prime}, \quad \hat{n}^{1}=\hat{n}, \\
\hat{n}^{2}=-\frac{1}{2} \hat{n}+\frac{\sqrt{3}}{2} \hat{n}^{\prime}, \quad \hat{n}^{3}=-\frac{1}{2} \hat{n}-\frac{\sqrt{3}}{2} \hat{n}^{\prime},
\end{gathered}
$$

where the sum is the sum of the Abelian directions of three $\mathrm{SU}(2)$ subgroups made of $\left(\hat{n}_{1}, \hat{n}_{2}, \hat{n}^{1}\right),\left(\hat{n}_{6}, \hat{n}_{7}, \hat{n}^{2}\right),\left(\hat{n}_{4},-\hat{n}_{5}, \hat{n}^{3}\right)$. Notice the factor $2 / 3$ in front of $\hat{A}_{\mu}^{p}$ in the $p$-summation. This is because the three $\mathrm{SU}(2)$ binding potentials are not independent.

From this we have the restricted the restricted QCD made of the restricted field strength,

$$
\mathcal{L}_{R C D}=-\sum_{p} \frac{1}{6}\left(\hat{F}_{\mu \nu}^{p}\right)^{2}
$$


which has the full SU(3) gauge symmetry.

With (11) we have the Abelian decomposition of the SU(3) gauge potential,

$$
\begin{gathered}
\vec{A}_{\mu}=\hat{A}_{\mu}+\vec{X}_{\mu}=\sum_{p}\left(\frac{2}{3} \hat{A}_{\mu}^{p}+\vec{W}_{\mu}^{p}\right), \\
\vec{X}_{\mu}=\sum_{p} \vec{W}_{\mu}^{p}, \\
\vec{W}_{\mu}^{1}=X_{\mu}^{1} \hat{n}_{1}+X_{\mu}^{2} \hat{n}_{2}, \quad \vec{W}_{\mu}^{2}=X_{\mu}^{6} \hat{n}_{6}+X_{\mu}^{7} \hat{n}_{7}, \\
\vec{W}_{\mu}^{3}=X_{\mu}^{4} \hat{n}_{4}-X_{\mu}^{5} \hat{n}_{5} .
\end{gathered}
$$

Here again $\vec{X}_{\mu}$ transforms covariantly, and can be decomposed to the three valence gluons $\vec{W}_{\mu}^{p}$ of the SU(2) subgroups. But unlike $\hat{A}_{\mu}^{p}$, they are mutually independent. So we have two binding gluons and six (or three complex) valence gluons in SU(3) QCD.

From (13) we have

$$
\begin{gathered}
\hat{D}_{\mu} \vec{X}_{v}=\sum_{p} \hat{D}_{\mu}^{p} \vec{W}_{v}^{p}, \quad \hat{D}_{\mu}^{p}=\partial_{\mu}+g \hat{A}_{\mu}^{p} \times, \\
\vec{X}_{\mu} \times \vec{X}_{v}=\sum_{p, q} \vec{W}_{\mu}^{p} \times \vec{W}_{v}^{q}, \\
\vec{F}_{\mu \nu}=\hat{F}_{\mu v}+\hat{D}_{\mu} \vec{X}_{v}-\hat{D}_{v} \vec{X}_{\mu}+g \vec{X}_{\mu} \times \vec{X}_{v} \\
=\sum_{p}\left[\frac{2}{3} \hat{F}_{\mu \nu}^{p}+\left(\hat{D}_{\mu}^{p} \vec{W}_{v}^{p}-\hat{D}_{\mu}^{p} \vec{W}_{v}^{p}\right)\right] \\
+\sum_{p, q} \vec{W}_{\mu}^{p} \times \vec{W}_{v}^{q},
\end{gathered}
$$

so that we have the following form of $\operatorname{SU}(3) \operatorname{ECD}[18,19]$

$$
\begin{gathered}
\mathcal{L}==\sum_{p}\left\{-\frac{1}{6}\left(\hat{F}_{\mu \nu}^{p}\right)^{2}-\frac{1}{4}\left(\hat{D}_{\mu}^{p} \vec{W}_{v}^{p}-\hat{D}_{\nu}^{p} \vec{W}_{\mu}^{p}\right)^{2}\right. \\
\left.-\frac{g}{2} \hat{F}_{\mu \nu}^{p} \cdot\left(\vec{W}_{\mu}^{p} \times \vec{W}_{v}^{p}\right)\right\}-\sum_{p, q} \frac{g^{2}}{4}\left(\vec{W}_{\mu}^{p} \times \vec{W}_{\mu}^{q}\right)^{2} \\
-\sum_{p, q, r} \frac{g}{2}\left(\hat{D}_{\mu}^{p} \vec{W}_{v}^{p}-\hat{D}_{\nu}^{p} \vec{W}_{\mu}^{p}\right) \cdot\left(\vec{W}_{\mu}^{q} \times \vec{W}_{\mu}^{r}\right) \\
-\sum_{p \neq q} \frac{g^{2}}{4}\left(\left(\vec{W}_{\mu}^{p} \times \vec{W}_{\nu}^{q}\right) \cdot\left(\vec{W}_{\mu}^{q} \times \vec{W}_{v}^{p}\right)\right. \\
\left.+\left(\vec{W}_{\mu}^{p} \times \vec{W}_{\nu}^{p}\right) \cdot\left(\vec{W}_{\mu}^{q} \times \vec{W}_{v}^{q}\right)\right) .
\end{gathered}
$$

This shows that the interactions in SU(3) QCD is more complicated than the SU(2) QCD.

But what is remarkable about (15) is that it is Weyl symmetric, symmetric under the permutationof the three SU(2) subgroups of SU(3). As we will see, this is very important for us to calculate the effective action of SU(3) QCD, because this allows us to express the SU(3) QCD effective action directly from the $\mathrm{SU}(2)$ result. 


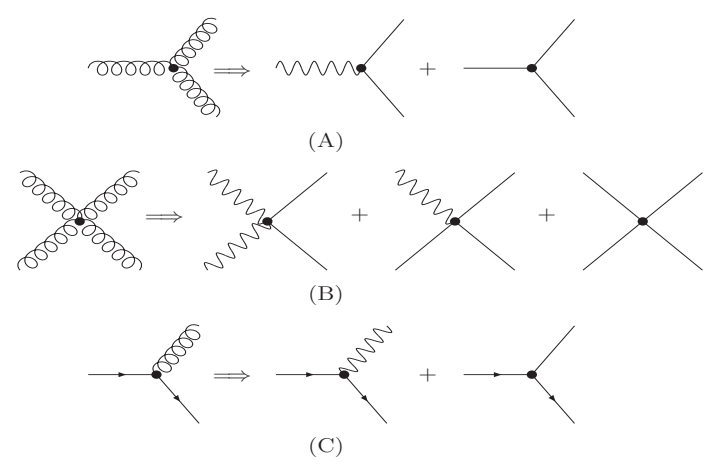

Figure 2. The decomposition of vertices in SU(3) QCD. The three and four point gluon vertices are decomposed in (A) and (B), and the quark gluon verteces are decomposed in (C). Notice that here (and in the followings) the neurons are representedby wiggly lines and the chromons are represented by straight lines.

We can easily add quarks in the Abelian decomposition,

$$
\begin{gathered}
\mathcal{L}_{q}=\sum_{k} \bar{\Psi}_{k}\left(i \gamma^{\mu} D_{\mu}-m\right) \Psi_{k} \\
=\sum_{k}\left[\bar{\Psi}_{k}\left(i \gamma^{\mu} \hat{D}_{\mu}-m\right) \Psi_{k}+\frac{g}{2} \vec{X}_{\mu} \cdot \bar{\Psi}_{k}\left(\gamma^{\mu} \vec{t}\right) \Psi_{k}\right] \\
=\sum_{p, k}\left[\bar{\Psi}_{k}^{p}\left(i \gamma^{\mu} \hat{D}_{\mu}^{p}-m\right) \Psi_{k}^{p}+\frac{g}{2} \vec{W}_{\mu}^{p} \cdot \bar{\Psi}_{k}^{p}\left(\gamma^{\mu} \vec{\tau}^{p}\right) \Psi_{k}^{p}\right], \\
\hat{D}_{\mu}=\partial_{\mu}+\frac{g}{2 i} \vec{t} \cdot \hat{A}_{\mu}, \quad \hat{D}_{\mu}^{p}=\partial_{\mu}+\frac{g}{2 i} \vec{\tau}^{p} \cdot \hat{A}_{\mu}^{p},
\end{gathered}
$$

where $m$ is the mass, $k$ and $p$ denote the flavor and color of the quarks, and $\Psi_{k}^{p}$ represents the three SU(2) quark doublets (i.e., $(r, b),(b, g)$, and $(g, r)$ doublets) of the $(r, b, g)$ quark triplet. With this the colors of the six chromons are given by $(r \bar{b}, b \bar{g}, g \bar{r}, \bar{r} b, \bar{b} g, \bar{g} r)$, which can be denoted by $(R, B, G, \bar{R}, \bar{B}, \bar{G})$.

We can show how the Abelian decomposition refines QCD interaction graphically. This is shown in Fig. 2. In (A) the three-point QCD gluon vertex is decomposed to two vertices made of one neuron and two chromons and three chromons. In (B) the four-point gluon vertex is decomposed to three vertices made of one neuron and three chromons, two neurons and two chromons, and four chromons. In (C) the quark-gluon vertex is decomposed to the quark-neuron vertex and quark-chromon vertex.

Notice that here (and in the following figures) the neurons are expressed by the wiggly lines (Maxwell part). This is because the monopole potential (Dirac part) makes the condensation, so that in the perturbative regime (inside the hadrons) it does not contribute to the Feynman diagrams. Also here three-point vertex made of three neurons or two neurons and one chromon, and four-point vertex made of three or four neurons are forbidden by the conservation of color. Moreover, the quark-neuron interaction does not change the quark color, but the quark-chromon interaction changes the quark color.

An important implication of Fig. 2 is that there are two types of gluon jets, the neuron jet and chromon jet. In principle we can test this experimentally by studying the gluon jets. Experiments can tell the difference between the photon-quark jet from the gluon-quark jet. If so, by (re-)analyzing the 
gluon-gluon jets and/or gluon-quark jets more carefully we could confirm that indeed there are two types of gluon jets. This can endorse the existence of two types of gluons.

But what is the most important is that this Abelian decomposition is gauge independent. This is because the decomposition is made without the gauge fixing. This has deep implications. The conventional wisdom is that all gluons are equal because of the gauge invariance, so that one can not differentiate the neutral gluons from the colored ones. This is simply not true. There is a mathematically well defined way to separate the neutral gluons from the colored ones gauge independently.

\section{Abelian Dominance and Monopole Condensation in QCD}

Our analysis tells that, although the Abelian decomposition does not change QCD, it makes many hidden structures of QCD explicit. First, it tells that RCD is responsible for the confinement, because the valence gluons (being colored) have to be confined $[4,13]$. This is the Abelian dominance advocated by 'tHooft [4].

But actually the Abelian domonance does not tell what is responsible for the confinement, because the restricted potential is made of two parts, the non-topological Maxwellian potential and topological Diracian potential. The Abelian decomposition tells exactly what is responsible for the confinement [13].

It allows us to prove that the monopole is responsible for the area law in the Wilson loop integral. Implementing (9) on lattice, we can performed a truly gauge independent lattice calculation and show that the monopole is responsible for the confinement. Two lattice groups, KEK-Chiba and SNUKonkuk, made the numerical calculation independently and confirmed the monopole domonance [20, 21]. The SNU-Konkuk result is shown in Fig. 3, which shows that the full gauge potential, the restricted potential, and the monopole potential all produce the same linear confining potential in Wilson loop integral. This assures that we only need the monopole potential for the confining force.

Second, it reduces the complicated non-Abelian gauge symmetry to a simple discrete symmetry called the color reflection invariance. To see this, consider the rotation of basis called the color reflection in SU(2) QCD

$$
\left(\hat{n}_{1}, \hat{n}_{2}, \hat{n}\right) \rightarrow\left(\hat{n}_{1},-\hat{n}_{2},-\hat{n}\right)
$$

Obviously this is a gauge transformation, so that this must remain a symmetry of QCD. On the other hand, the isometry condition (1) does not change under (17). This means that, after we select the Abelian direction $\hat{n}$ we have two different but gauge equivalent Abelian decompositions related by the color reflection.

What makes the color reflection symmetry so important is that it is the only remaining symmetry of the full gauge symmetry left over, after we make the Abelian decomposition [2,3]. So the color reflection invariance plays the role of the non-Abelian gauge invariance after we have chosen the Abelian direction. This greatly simplifies for us to implement the gauge invariance to calculate the QCD effective action [18, 19].

The color reflection symmetry of SU(2) QCD is made of 4 elements, but in SU(3) QCD it consists of 24 element. An important subgroup of the color reflection symmetry is the Weyl symmetry, which is made of the permutation of the colors. This automatically guarantees that QCD is invariant under the permutation of any two colors. In SU(2) QCD we have two colors so that the Weyl symmetry 


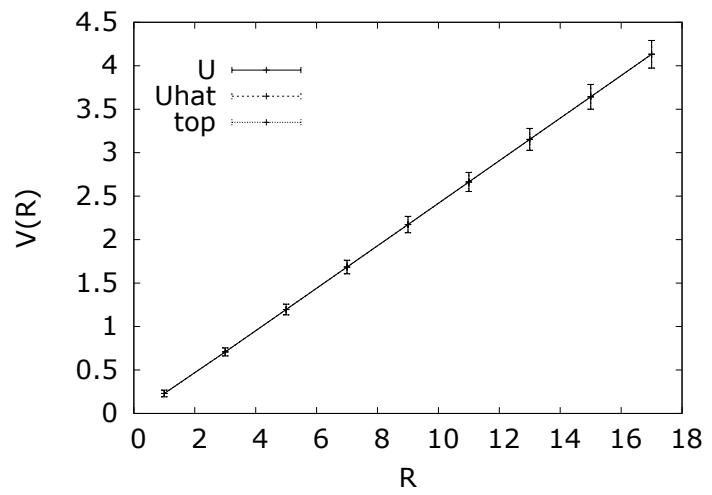

Figure 3. The lattice QCD calculation which establishes the monopole dominance in Wilson loop.

is made of two elements. But in SU(3) QCD it is made of six elements since SU(3) QCD has three colors.

Moreover, the Abelian decomposition automatically puts QCD to the background field formalism [22-24]. This is because we can view the Abelian part as the classical background and the colored part as the quantum fluctuation. This enlarges and doubles the gauge symmetry to the classical and quantum gauge symmetries, since we can associate the original gauge symmetry to the classical part or to the quantum part.

More importantly this allows us to calculate the effective action of QCD and demonstrate the monopole condensation gauge independently. Savvidy was the first who tried to prove the monopole condensation calculating the effective action of SU(2) QCD, and has "almost" succeeded to do so [7]. However, this monopole condensation called the Savvidy vacuum turned out to be unstable, which is known as the Savvidy-Nielsen-Olesen (SNO) instability [8]. Since then many people tried to restore the stability without much success $[9,10]$.

Actually there are ways to restore the stability in the Savvidy vacuum [25, 26]. But the real problem with the Savvidy vacuum is not that it is unstable but that the gauge invariance was not properly implemented in the calculation [18]. First of all, the Savvidy background was not the monopole background. At that time people did not know how to separate the monopole background gauge independently. But most seriously, the calculation of the functional determinant of the chromons was not done gauge invariantly, so that the determinant contained the tachyonic mode which made the Savvidy vacuum unstable. This was the critical defect.

The Abelian decomposition allows us to calculate the effective action of QCD gauge independently and gauge invariantly. First, the Abelian decomposition decomposes the gauge potential to the binding potential and the valence potential gauge independently. Second, it separates the monopole part from the binding potential gauge independently. This allows us to separate the monopole background gauge independently. Without the Abelian decomposition this would have been impossible.

Third, in the earlier calculations it was not clear which gluons are integrated out in the functional integral. As a result it was not clear what kind of effective action was calculated. But with the Abelian decomposition we can clearly say that, integrating out only the gauge covariant chromons, we are calculating the effective action of RCD. 
Most importantly, imposing the color reflection invariance in the calculation of the functional determinant of the chromons under the monopole background, we can remove the tachyonic mode which cures the SNO instability.

\section{QCD Effective Action and Monopole Condensation}

To show how we can do this we start from the SU(2) QCD Lagrangian (8) and fix the quantum gauge by the generalized Lorentz gauge condition

$$
\vec{F}=\hat{D}_{\mu} \vec{X}_{\mu}=0, \quad \mathcal{L}_{g f}=-\frac{1}{2 \xi}\left(\hat{D}_{\mu} \vec{X}_{\mu}\right)^{2}
$$

The corresponding Faddeev-Popov determinant can be expressed by

$$
\begin{gathered}
M_{a b}^{F P}=\frac{\delta F_{a}}{\delta \alpha^{b}}=\Pi_{a c}\left(\hat{D}_{\mu} D_{\mu}\right)_{c b}, \\
\Pi_{a b}=\delta_{a b}-\hat{n}_{a} \hat{n}_{b} .
\end{gathered}
$$

Notice that $\Pi_{a b}$ is the projection operator which projects out the $\hat{n}$ component.

To proceed we have to choose the background. We choose the gauge independent and parity conserving arbitrary constant Diracian background,

$$
\hat{F}_{\mu \nu}^{(b)}=\bar{H}_{\mu \nu} \hat{n}
$$

Notice that when $\bar{H}_{\mu v}=H \delta_{[m}^{1} \delta_{n]}^{2}$, it describes the monopole background. In comparison Savvidy background was the parity violating Maxwellian $\bar{F}_{\mu \nu} \hat{n}$. This is because the background was chosen without the Abelian decomposition, so that there was no way to distinguish the topological monopole background with the non-topological magnetic background.

With this we can calculate the effective action of RCD integrating out the valence potential [18]

$$
\begin{gathered}
\exp \left[i S_{e f f}\left(\hat{A}_{\mu}\right)\right]=\int \mathcal{D} \vec{X}_{\mu} \mathcal{D} \vec{c} \mathcal{D} \vec{c}^{*} \\
\exp \left\{i \int \left[-\frac{1}{4} \hat{F}_{\mu \nu}^{2}-\frac{1}{4}\left(\hat{D}_{\mu} \vec{X}_{v}-\hat{D}_{v} \vec{X}_{\mu}\right)^{2}\right.\right. \\
-\frac{g}{2} \hat{F}_{\mu v} \cdot\left(\vec{X}_{\mu} \times \vec{X}_{v}\right)-\frac{g^{2}}{4}\left(\vec{X}_{\mu} \times \vec{X}_{v}\right)^{2} \\
\left.\left.-\frac{1}{2 \xi}\left(\hat{D}_{\mu} \vec{X}_{\mu}\right)^{2}+\vec{c}^{*} \hat{D}_{\mu} D_{\mu} \vec{c}\right] d^{4} x\right\},
\end{gathered}
$$

where $\vec{c}$ and $\vec{c}^{*}$ are the ghost fields which are orthogonal to $\hat{n}$. Here we need only the ghosts which are orthogonal to $\hat{n}$ because they come from the gauge fixing of the valence gluons which are orthogonal to $\hat{n}$.

Integrating out the valence potential under the constant Diracian background imposing the color reflection invariance (the C-projection) in the calculation of the functional determinant of the chromons, 
we obtain [18]

$$
\begin{gathered}
\Delta \mathcal{L}=\lim _{\epsilon \rightarrow 0} \frac{1}{8 \pi^{2}} \int_{0}^{\infty} \frac{d t}{t^{3-\epsilon}} \frac{a b t^{2} / \mu^{4}}{\sinh \left(a t / \mu^{2}\right) \sin \left(b t / \mu^{2}\right)} \\
\times\left[\exp \left(-2 a t / \mu^{2}\right)+\exp \left(+2 i b t / \mu^{2}\right)-1\right], \\
a=\frac{g}{2} \sqrt{\sqrt{\bar{H}^{4}+(\bar{H} \tilde{H})^{2}}+\bar{H}^{2}} \\
b=\frac{g}{2} \sqrt{\sqrt{\bar{H}^{4}+(\bar{H} \tilde{H})^{2}}-\bar{H}^{2}} .
\end{gathered}
$$

This is the new integral expression of QCD effective action. Notice that $a$ describes the Diracian magnetic (i.e., monopole) background, but $b$ describes Diracian electric background.

We emphasize that here the C-projection plays exactly the same role as the G-parity in string theory. In NSR string the GSO projection restores the supersymmetry and modular invariance by projecting out the tachyonic vacuum $[11,12]$. Just like the G-projection, the C-projection in $Q C D$ removes the tachyonic modes and restores the gauge invariance of the effective action.

From this we have [18]

$$
\mathcal{L}_{e f f}= \begin{cases}-\frac{a^{2}}{2 g^{2}}-\frac{11 a^{2}}{48 \pi^{2}}\left(\ln \frac{a}{\mu^{2}}-c^{\prime}\right), & b=0 \\ \frac{b^{2}}{2 g^{2}}+\frac{11 b^{2}}{48 \pi^{2}}\left(\ln \frac{b}{\mu^{2}}-c^{\prime}\right) & \\ -i \frac{11 b^{2}}{96 \pi}, & a=0\end{cases}
$$

The effective action (22) generates the much desired dynamical symmetry breaking and the dimensional transmutation in QCD. To demonstrate this notice that, with $b=0,(22)$ provides the following effective potential

$$
V=\frac{a^{2}}{2 g^{2}}\left[1+\frac{11 g^{2}}{24 \pi^{2}}\left(\ln \frac{a}{\mu^{2}}-c^{\prime}\right)\right] .
$$

Defining the running coupling $\bar{g}$ by $[25,26]$

$$
\left.\frac{\partial^{2} V}{\partial a^{2}}\right|_{a=\bar{\mu}^{2}}=\frac{1}{\bar{g}^{2}}
$$

we have

$$
\begin{gathered}
\frac{1}{\bar{g}^{2}}=\frac{1}{g^{2}}+\frac{11}{24 \pi^{2}}\left(\ln \frac{\bar{\mu}^{2}}{\mu^{2}}-c^{\prime}+\frac{3}{2}\right), \\
\beta(\bar{g})=\bar{\mu} \frac{\partial \bar{g}}{\partial \bar{\mu}}=-\frac{11 \bar{g}^{3}}{24 \pi^{2}} .
\end{gathered}
$$

This is exactly the same $\beta$-function in perturbative $\mathrm{SU}(2) \mathrm{QCD}$ which assures the asymptotic freedom [27]. 


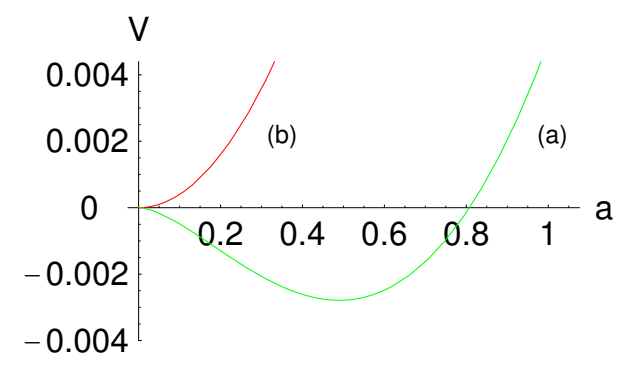

Figure 4. The effective potential of SU(2) QCD in the monopole background. Here (a) is the effective potential and (b) is the classical potential.

In terms of the running coupling the renormalized potential is given by [18]

$$
V_{\text {ren }}=\frac{a^{2}}{2 \bar{g}^{2}}\left[1+\frac{11 \bar{g}^{2}}{24 \pi^{2}}\left(\ln \frac{a}{\bar{\mu}^{2}}-\frac{3}{2}\right)\right],
$$

which generates a non-trivial local minimum at

$$
\langle a\rangle=\bar{\mu}^{2} \exp \left(-\frac{24 \pi^{2}}{11 \bar{g}^{2}}+1\right) .
$$

This is nothing but the dimensional transmutation by the monopole condensation. The corresponding effective potential is plotted in Fig. 4, where we have assumed $\bar{\alpha}_{s}=1$ and $\bar{\mu}^{2}=1$. This should be compared with the old calculation of the QCD effective action [7-10].

At the first glance the calculation of the SU(3) QCD effective action looks much more complicated. Fortunately, we can use the Weyl symmetry of SU(3) ECD (15) to calculate the effective action. Since the SU(3) QCD is the Weyl symmetric linear combination of SU(2) QCD, we can easily generalize the above $\mathrm{SU}(2)$ result to $\mathrm{SU}(3) \mathrm{QCD}$. We have

$$
\mathcal{L}_{e f f}= \begin{cases}-\sum_{p}\left(\frac{a_{p}^{2}}{3 g^{2}}+\frac{11 a_{p}^{2}}{48 \pi^{2}}\left(\ln \frac{a_{p}}{\mu^{2}}-c\right)\right), \quad b=0 \\ \sum_{p}\left(\frac{b_{p}^{2}}{3 g^{2}}+\frac{11 b_{p}^{2}}{48 \pi^{2}}\left(\ln \frac{b_{p}}{\mu^{2}}-c\right)\right. & \\ \left.-i \frac{11 b_{p}^{2}}{96 \pi}\right), & a=0\end{cases}
$$

where $a_{p}(p=1,2,3)$ and $b_{p}$ are the Diracian chromomagnetic (i.e., monopole) and chromoelectric background of three SU(2) subgroups. Notice that, although the background is fixed by two parameters $\left(a_{3}\right.$ and $a_{8}$ or $b_{3}$ and $\left.b_{8}\right)$, the effective action depends on three parameters $a_{p}$ or $b_{p}$.

It should be emphasized that, without the Weyl symmetry, we could not have obtained the above rsult. It is the Weyl symmetry that allows us to obtain the effective action of SU(3) QCD without calculation. In general the Weyl symmetry exists in $\mathrm{SU}(\mathrm{N}) \mathrm{QCD}$, which means that as far as the confinement is concerned SU(N) QCD is essentially the same as SU(2) QCD. This is the power of the Weyl symmetry. 


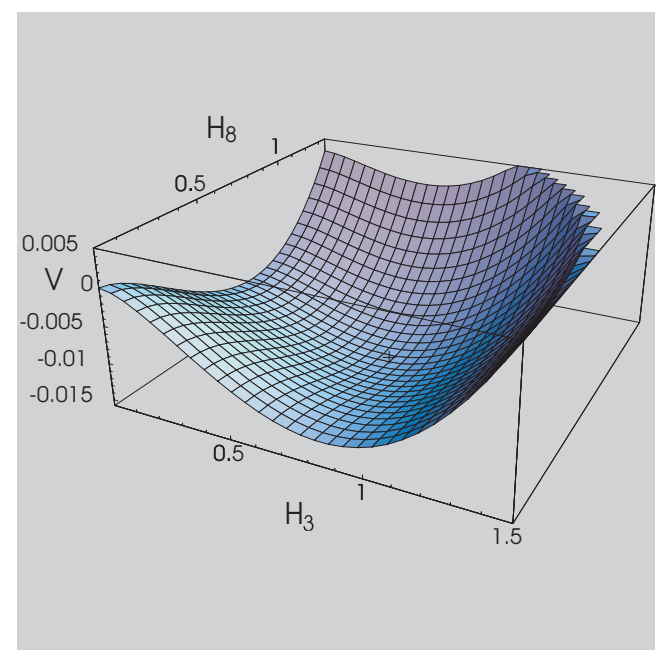

Figure 5. The effective potential of SU(3) QCD.

With $b_{p}=0$, we can easily show that the effective potential (29) has (after the renormalization) the true minimum at

$$
\left\langle a_{1}\right\rangle=\left\langle a_{2}\right\rangle=\left\langle a_{3}\right\rangle=\bar{\mu}^{2} \exp \left(-\frac{16 \pi^{2}}{11 g^{2}}+\frac{3}{4}\right) .
$$

The effective potential is shown in Fig. 5. Notice that the vacuum is Weyl symmetric, which is natural. This demonstrates the monopole condensation which generates the desired mass gap in SU(3) QCD.

The effective action has two important features. First, it is invariant under the dual transformation $[25,26]$

$$
a \rightarrow-i b, \quad b \rightarrow i a,
$$

This duality was first discovered in the QED effective action [28]. But subsequently this duality has been shown to be a fundamental symmetry of the effective action of gauge theory, Abelian and non-Abelian.

Another important feature of (22) and (29) is that when $a=0$ (or $a_{p}=0$ ) the imaginary part has a negative signature. This implies the pair annihilation of chromons in chromoelectric background $[18,19,29]$. This must be contrasted with the QED effective action where the electron loop integral generates a positive imaginary part $[28,30]$. The positive imaginary part in QED means the pair creation which generates the screening. On the other hand in QCD we have the negative imaginary part, and thus the pair annihilation of chromons.

This is closely related to the asymptotic freedom (anti-screening) of gluons. In QCD it is well known that the gluons and quarks play opposite roles in the asymptotic freedom [27]. In other words the quarks enhance the screening while the gluons overide the quarks and diminish it to generate the anti-screening. We can understand this with the pair creation of the quarks and the pair annihilation of 
the chromons by the chromo-electric field $[18,19]$. And the QCD effective action confirms this whith the negative imaginariy part.

\section{Discussions}

In this talk we have discussed the importance of the Abelian decomposition in $\mathrm{QCD}$, and show how the Abelian decomposition allows us to demonstrate the monopole copndensation. The Abelian decomposition simplifies QCD dynamics greatly. It tells that QCD has two gluons which play different roles. Perturbatively (in terms of the Feynman diagrams) the neurons play the role of the photon and the chromons play the role of (massless) charged vector fields in QED. Non-perturbatively, however, (the monopole part of) the neurons become the confining agents. In contrast, the chromons become the confined prisoners. Without the Abelian decomposition we can not tell this difference because all gluons are treated on equal footing.

The Abelian decomposition tells that it is the monopole which confines the color. This has been proved in lattice QCD. Implementing the Abelian decomposition in lattice we have shown that it is the monopole potential which confines the color. Moreover, the Abelian decomposition allows us to calculate the QCD effective potential gauge independently, and demonstrate the monopole condensation in QCD explicitly.

The monopole condensation generates the dynamical symmetry breaking and dimensional transmutation (the generation of the mass gap) which in turn confines the color. But we emphasize that this confinement mechanism is not exactly the color confinement by the dual Meissner effect. There are important differences between the two mechanisms.

In superconductor the supercurrent created by the Cooper pair confines (more precisely shields) the magnetic field. This is the Meissner effect. The dual Meissner effect in QCD proposes that the monopole-antimonopole pair condensation could play the role of the Cooper pair and confine the chromoelectric fiield in QCD. In detail, however, this view does not hold.

First, in superconductor the Cooper pair has electric charge, but in QCD the Weyl symmetry tells that the monopole is gauge equivalent with the anti-monopole. This means that the monopoleantimonopole pair has no chromomagnetic charge. Second, in superconductor the magnetic field is generated by the electric current. But in QCD the chromoelectric field does not come from the monopole current. It is created by the color charge.

Third, in superconductor the supercurrent generated by the Cooper pairs shields the magnetic field. But in QCD the monopole condensation (more precisely the monopole-antimonopole psair condensation) confines the chromoelectric field. Fourth, in superconductor the Higgs mechanism breaks the symmetry. But in QCD the symmetry is broken dynamically. So the two confining mechanism is not exactly dual to each other.

An important question at this point is what is the consequence of the monopole condensation other than the confinement, if there is any. This is a very important question, because in the Higgs mechanism we have the $0^{++}$scalar mode (the Cooper pair) and the $1^{--}$spin-one mode whose masses provides the corelation length of the Cooper pair and the penetration length of the magnetic field.

Can the monopole condensation generate similar modes? Although we have pointed out that the monopole condensation is different from the dual Meissner effect, the monopole condensation also creates two scales, the pair correlation length and the chromoelectric flux penetration length. And 
the QCD vacuum made of the monopole condensation can have the vacuum fluctuation, which may generated the fluctuation mode. And this could be identified as the $0^{++}$scalar mode, whose mass can be identified as the QCD mass scale $\Lambda_{Q C D}$. So it is natural to suppose that the monopole condensation generates an observable result other than the confinement, which can be verified by experiment.

The remaining question is do we have the spin-one mode? In principle, the correlation length and the penetration length in QCD could be different, but so far the general wisdom is that there is only one QCD scale. This implies that the two scales in QCD are in fact the same, which implies that unlike in the superconductor QCD may not have the spin-one mode.

The above discussion strongly suggest that the monopole condensation has an important observable consequence, the apperance of the scalar vacuum fluctuation mode. And this mode is totally different from all the other hadrons. So it is very important to check the existence of such mode. We suggest that $f_{0}(500)$ could be such a mode [31].

On the other hand there is also a possibility that the QCD vacuum, i.e., the monopole condensation could be like the Dirac sea, the QED vacuum. Notice that the Dirac sea made of the electro-positron pairs has no vacuum fluctuation mode. If the QCD vacuum is like the Dirac sea, we will have no vacuum fluctuation mode at all in QCD. This makes the experimental confirmation of the vacuum fluctuation mode in QCD an urgent issue.

Before we close we emphasize that the Abelian decomposition is not just a theoretical proposition. It can be tested by experiments. A simplest way is to do so is to confirm the existence of two different gluon jets. Clearly the neuron jets, being color neutral are expected to behave like the photon jet. On the other hand the chromon jets, being colored should behave like the quark jet. So, in principle we can differenciate them and confirm the existence of two gluons experimentally.

Moreover, we can test it experimentally studying the hadron spectroscopy. This is because the Abelian decomposition generalizes the quark model to the quark and chromon model. The reason is that the chromons, just like the quarks, become the gauge covariant colored source which can be viewed as the colored constituents of hadrons [31].

So we can construct new hybrid hadronic states with quarks and chromons. Moreover, we can construct the glueballs made of chromons. And these new hadronic states could be verified by experiments. This tells that we can actually test the Abelian decomposition experimentally without ambiguity. The details of the Abelian decomposition and its applications will be published elsewhere [32].

\section{ACKNOWLEDGEMENT}

The work is supported in part by the Korean National Research Foundation funded by the Ministry of Education (Grant 2015-R1D1A1A0-1057578) and by Center for Quantum Space-time at Sogang University.

\section{References}

[1] Y. Nambu, Phys. Rev. D10, 4262 (1974); S. Mandelstam, Phys. Rep. 23C, 245 (1976); A. Polyakov, Nucl. Phys. B120, 429 (1977).

[2] Y. M. Cho, Phys. Rev. D21, 1080 (1980). See also Y. S. Duan and M. L. Ge, Sci. Sinica 11,1072 (1979).

[3] Y. M. Cho, Phys. Rev. Lett. 46, 302 (1981); Phys. Rev. D23, 2415 (1981). 
[4] G. 't Hooft, Nucl. Phys. B190, 455 (1981).

[5] A Kronfeld, G. Schierholz, and U. Wiese, Nucl. Phys. B293, 461 (1987); A Kronfeld, M. Laursen, G. Schierholz, and U. Wiese, Phys. Lett. B198, 516 (1987); T. Suzuki and I. Yotsuyanagi, Phys. Rev. D42, 4257 (1990).

[6] S. Coleman and E. Weinberg, Phys. Rev. D7, 1888 (1973).

[7] G. K. Savvidy, Phys. Lett. B71, 133 (1977).

[8] N. Nielsen and P. Olesen, Nucl. Phys. B144, 485 (1978); B160, 380 (1979); C. Rajiadakos, Phys. Lett. B100, 471 (1981).

[9] W. Dittrich and M. Reuter, Phys. Lett. B128, 321, (1983); B144, 99 (1984); M. Reuter, M. G. Schmidt, and C. Schubert, Ann. Phys. 259, 313 (1997).

[10] A. Yildiz and P. Cox, Phys. Rev. D21, 1095 (1980); M. Claudson, A. Yilditz, and P. Cox, Phys. Rev. D22, 2022 (1980); J. Ambjorn and R. Hughes, Phys. Lett. B113, 305 (1982).

[11] F. Gliozzi, J. Scherk, and D. Olive, Nucl. Phys. B122, 253 (1977).

[12] See, e. g., M. Green, J. Schwarz, and E. Witten, Superstring Theory Vol. I (Cambridge University Press) 1987; M. Kaku, Introduction to Superstrings (Springer-Verlag) 1988.

[13] Y. M. Cho, Phys. Rev. D62, 074009 (2000).

[14] L. Faddeev and A. Niemi, Phys. Rev. Lett. 82, 1624 (1999).

[15] S. Shabanov, Phys. Lett. B458, 322 (1999); B463, 263 (1999); H. Gies, Phys. Rev. D63, 125023 (2001).

[16] R. Zucchini, Int. J. Geom. Meth. Mod. Phys. 1, 813 (2004).

[17] Y. M. Cho, Phys. Rev. Lett. 44, 1115 (1980).

[18] Y. M. Cho, Franklin H. Cho, and J. H. Yoon, Phys. Rev. D87, 085025 (2013).

[19] Y. M. Cho, Int. J. Mod. Phys. A29, 1450013 (2014).

[20] S. Kato, K. Kondo, T. Murakami, A. Shibata, T. Shinohara, and S. Ito, Phys. Lett. B632, 326 (2006); B645, 67 (2007); B653, 101 (2007); B669, 107 (2008).

[21] N. Cundy, Y. M. Cho, W. Lee, and J. Leem, Phys. Lett. B729, 192 (2014); N. Cundy, Y. M. Cho, W. Lee, and J. Leem, Nucl. Phys. B895, 64 (2015).

[22] B. de Witt, Phys. Rev. 162, 1195 (1967); 1239 (1967).

[23] See for example, C. Itzikson and J. Zuber, Quantum Field Theory (McGraw-Hill) 1985; M. Peskin and D. Schroeder, An Introduction to Quantum Field Theory (Addison-Wesley) 1996.

[24] W. S. Bae, Y. M. Cho, and S. W. Kim, Phys. Rev. D65, 025005 (2001).

[25] Y. M. Cho and D. G. Pak, Phys. Rev. D65, 074027 (2002); Y. M. Cho, H. W. Lee, and D. G. Pak, Phys. Lett. B525, 347 (2002).

[26] Y. M. Cho, M. L. Walker, and D. G. Pak, JHEP 05, 073 (2004); Y. M. Cho and M. L. Walker, Mod. Phys. Lett A19, 2707 (2004).

[27] D. Gross and F. Wilczek, Phys. Rev. Lett. 30, 1343 (1973); H. Politzer, Phys. Rev. Lett. 30, 1346 (1973).

[28] Y. M. Cho and D. G. Pak, Phys. Rev. Lett. 86, 1947 (2001); 91, 039151 (2003); W. S. Bae, Y. M. Cho, and D. G. Pak, Phys. Rev. D64, 017303 (2001).

[29] V. Schanbacher, Phys. Rev. D26, 489 (1982).

[30] J. Schwinger, Phys. Rev. 82, 664 (1951).

[31] Y. M. Cho, X. Y. Pham, Pengming Zhang, Ju-Jun Xie, and Li-Ping Zou, Phys. Rev. D91, 114020 (2015).

[32] Y. M. Cho, to be published. 